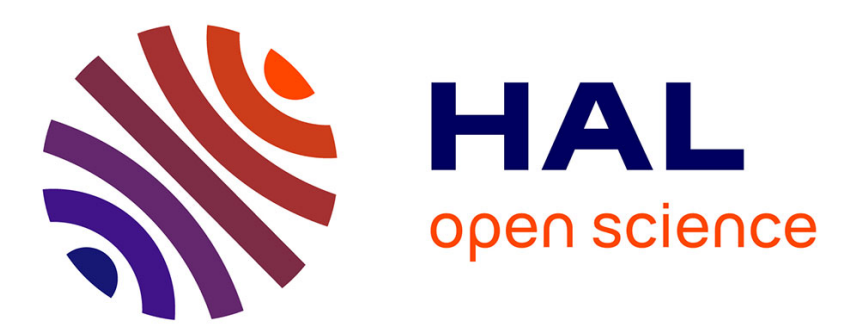

\title{
Fast myopic 2D-SIM Super Resolution Microscopy with Joint Modulation Pattern Estimation
}

\author{
François Orieux, Vincent Loriette, Jean-Christophe Olivo-Marin, Eduardo
} Sepulveda, Alexandra Fragola

\section{- To cite this version:}

François Orieux, Vincent Loriette, Jean-Christophe Olivo-Marin, Eduardo Sepulveda, Alexandra Fragola. Fast myopic 2D-SIM Super Resolution Microscopy with Joint Modulation Pattern Estimation. Inverse Problems, 2017, 33 (12), pp.125005. 10.1088/1361-6420/aa8f5b . hal-01623761

\section{HAL Id: hal-01623761 https://hal.science/hal-01623761}

Submitted on 5 Mar 2020

HAL is a multi-disciplinary open access archive for the deposit and dissemination of scientific research documents, whether they are published or not. The documents may come from teaching and research institutions in France or abroad, or from public or private research centers.
L'archive ouverte pluridisciplinaire HAL, est destinée au dépôt et à la diffusion de documents scientifiques de niveau recherche, publiés ou non, émanant des établissements d'enseignement et de recherche français ou étrangers, des laboratoires publics ou privés. 


\title{
Fast myopic 2D-SIM Super Resolution Microscopy with Joint Modulation Pattern Estimation
}

\author{
François Orieux ${ }^{1}$, Vincent Loriette ${ }^{2}$, Jean-Christophe \\ Olivo-Marin ${ }^{3}$, Eduardo Sepulveda ${ }^{4}$, Alexandra Fragola ${ }^{2}$ \\ ${ }^{1}$ Laboratoire des Signaux et Systèmes (Univ. Paris-Sud - CNRS - CentraleSupélec - \\ Université Paris-Saclay), 3 rue Joliot-Curie, 91192 Gif-sur-Yvette, France \\ ${ }^{2}$ Laboratoire de physique et d'étude des matériaux (CNRS - UPMC - ESPCI), \\ 75005 Paris, France \\ ${ }^{3}$ Unité d'Analyse d'Images Biologiques (Institut Pasteur - CNRS), 75015 Paris, \\ France \\ ${ }^{4}$ Laboratoire de Physique Nucléaire et des Hautes Énergies (IN2P3 - CNRS - \\ UPMC - UPD), 75252 Paris, France \\ E-mail: orieux@12s.centralesupelec.fr
}

\begin{abstract}
Super-resolution in Structured Illumination Microscopy (SIM) is obtained through de-aliasing of modulated raw images, in which high frequencies are measured indirectly inside the optical transfer function. Usual approaches that use 9 or 15 images are often too slow for dynamic studies. Moreover, as experimental conditions change with time, modulation parameters must be estimated within the images. This paper tackles the problem of image reconstruction for fast super resolution in SIM, where the number of available raw images is reduced to four instead of nine or fifteen. Within an optimization framework, the solution is inferred via a joint myopic criterion for image and modulation (or acquisition) parameters, leading to what is frequently called a myopic or semi-blind inversion problem. The estimate is chosen as the minimizer of the nonlinear criterion, numerically calculated by means of a block coordinate optimization algorithm. The effectiveness of the proposed method is demonstrated for simulated and experimental examples. The results show precise estimation of the modulation parameters jointly with the reconstruction of the super resolution image. The method also shows its effectiveness for thick biological samples.
\end{abstract}


Fast myopic 2D-SIM Super Resolution Microscopy with Joint Modulation Pattern Estimation2

\section{Introduction}

Fluorescence microscopy, where only molecules marked by fluorophores are visible, is a fundamental tool of biology, but remains fundamentally limited in resolution by diffraction (often modeled by a filter in Fourier space). The last twenty years have seen numerous developments in super resolution fluorescence microscopy, enabling resolutions in the 10 to $100 \mathrm{~nm}$ using these techniques for dynamic studies remains a challenge as they require sample scanning or multiple image acquisitions. Localization super resolution methods, such as photo-activated localization microscopy (PALM) or stochastic optical reconstruction microscopy (STORM) [1], are based on the localization of individual, and supposedly separate, photoactivable fluorophores. Thousands of exposures are necessary to build the final high-resolution image, which strongly limits the usefulness of these techniques for live imaging. Stimulated emission depletion (STED) microscopy [2] provides nanometer resolution by reducing the diffraction spot size through stimulated emission. The reconstructed image is obtained by scanning the sample. Even though a recent publication has demonstrated the capability of parallelizing 2000 STED spots, 100 acquired images are still required to build the superresolution image [3].

An alternative approach used in structured illumination microscopy (SIM) consists of illuminating and imaging the entire field of view and using a limited amount of raw data acquisitions. Illumination by a sinusoidal fringe pattern makes high spatial frequencies of the sample response, previously filtered, appear inside the support of the transfer function. An algorithm, after measurement, reconstructs a high-resolution image by demodulation and Wiener filtering.

SIM has provided $[4,5,6]$ resolution down to $100 \mathrm{~nm}$ at a rate of $\approx 11 \mathrm{~Hz}$ and an $8 \mu \mathrm{m}^{2}$ field of view [7]. Recently, Betzig and coworkers [4] demonstrated nonlinear (with the presence of high-order modulation) SIM capability to reach a resolution of $62 \mathrm{~nm}$ in living cells. These performances paved the way towards high-resolution imaging in living samples where numerous biological processes require a subsecond temporal resolution. For living cells studies, TIRF illumination, where only a hundred nanometer thick slice of the sample is observed, is the most common in the literature $[4,5,6]$ but forbids observation inside the cell. Although SIM can be used for 3D samples, it requires at least 15 images $[4,5,6,7]$ per optical section.

Furthermore, the reconstruction may generate artifacts that often appear as residual modulation, especially with thick samples, if sufficient attention is not paid to estimation of the modulation parameters [8, 9, 5]. Schaefer et al. [8] present a detailed and precise analysis of possible artifacts as well as an algorithm based on the post analysis of the result to minimize it. Wicker et al. [5] propose an algorithm based on the weighted crosscorrelation between the central spectrum and the replication due to the modulation in Fourier space. On the contrary, the proposed approach does not depend on the possible patterns of artifacts but rather on data simulation and criterion fitting.

A major alternative to harmonic modulation in classical SIM is the Blind-SIM 
approach $[10,11,12,13]$. With the blind-SIM approach, a large number of images is generated with different illumination patterns created by speckle. The stochastic variation of the modulation allows to retrieve the high-resolution image. Very good results have been obtained and the technique is promising for robust reconstruction, despite the great number of images required. Ayuk et al. [12] adapted the technique to distorted modulations. However, currently, blind-SIM is not known to be able to perform nonlinear reconstruction. Unfortunately, Blind-SIM has not been validated on living biological samples yet. In our case, the distortion made by the sample does not seem to be the major limitation, and we have not further investigated this possibility, especially as we consider the existing problem of harmonic modulation.

A paper by Dong et al. [14] shows an algorithm to reconstruct the image with only four raw images. In comparison to the proposed approach, the reconstruction is defined by the algorithm without clear argumentation about which image is reconstructed. Criterion based methods instead model explicitly the information as presented in Sec. 3, and optimization algorithm is a tool to reach the minimum. As a consequence, the reconstruction is not really well-defined, and the stability of the alternative reconstruction step with the modulation will be hard to guarantee. Finally, contrary to the previous work [15], the proposed approach allows to simplify the optical setup and estimate the modulation parameters that were fixed in the above cited work. Another important distinction is the nature of the estimator and algorithm that were the posterior mean and Monte Carlo Markov Chain (MCMC) in [15].

This paper describes a new methodological framework and an original algorithm for 2D joint myopic estimation based on a criterion to minimize. Compared to previous techniques, the proposed approach focuses on harmonic modulation estimation within the original Gustafsson framework and small number of raw images. The image reconstructed cannot be better than that obtained with perfectly reconstructed classical SIM, or Blind-SIM or [15]. However, even if the image reconstructed compares directly to other approaches, the proposed approach introduces several improvements in the acquisition and reconstruction steps. The algorithm is derived from the estimator and criterion, whereas the usual approaches define their solutions by the algorithms. The first advantage of our proposed method, as already mentioned in [15], is that it only needs four raw images to reconstruct one super resolution image in linear SIM and eight raw images in nonlinear SIM. In addition, there is no limitation to the modulation pattern that can be arbitrary if a parameterized pattern is available (we study only harmonic patterns here). Second, our algorithm can estimate, along with the image, the modulation parameters that need to be estimated from the data, even with four images. The Shroff et al method [9] is the only other method, to the best of our knowledge, which can provide a phase parameter value when only four raw images are used. The cross-correlation proposed by Wicker et al. [5] provides a very good result but requires component separation and is presented for phase estimation only. The proposed algorithm also estimates frequency (or orientation) and contrast modulation, and with a much better accuracy than [9]. 
Fast myopic 2D-SIM Super Resolution Microscopy with Joint Modulation Pattern Estimation4

Finally, when fringes are generated by the interference of the first orders of a diffracted beam, our method allows the use of the zero (or more) order without constraints. In such cases, standard methods need at least fifteen images $[5,6,16]$. In contrast, our method only needs four images. This point is interesting for two more reasons: interference with zero order introduces a modulation at half the frequency of the main fringe, leading to a better SNR for parameter estimation, and the optical setup does not need to block the zero order.

The remainder of this paper is organized as follows. Section 2 shows the underlying principles of SIM and our proposed approach for image number reduction. Section 3 presents our data model and specific constraints on the image and modulation parameters allowing us to jointly estimate all the unknowns, that is all modulation parameters and pixels of the image. Section 4 is devoted to the joint optimizer algorithm and the final section 5 presents the results. Simulated examples are used to quantify the effectiveness of the proposed algorithm and results for experimental data with thick samples are presented.

\section{Amplitude modulation and redundancy}

The diffraction theory states that incoherent optical systems can be described in Fourier space with the optical transfer function (OTF) $H\left(\nu_{x}, \nu_{y}\right)$, which is theoretically equal to zero for all frequencies beyond the cut-off frequency $\nu_{c}$. All information outside this bound is lost, and the underlying idea of SIM is injecting high frequencies inside the support of the OTF, owing to amplitude modulation, before filtering.

Let us denote the original image $f(x, y) \in L_{2}$ and its continuous Fourier transform $F\left(\nu_{x}, \nu_{y}\right)$. In SIM the illumination pattern is considered to be, up to an amplitude factor,

$$
m(x, y)=1+\beta \cos \left(\pi k_{x} x+\pi k_{y} y+\phi\right)+
$$

$$
\alpha \cos \left(2 \pi k_{x} x+2 \pi k_{y} y+2 \phi\right) .
$$

The fringe at the $k_{x} / 2$ frequency comes from the interference between the zero order and the two orders \pm 1 , and the fringe at the $k_{x}$ frequency from the interference between the orders -1 and +1 . The parameters $\alpha$ and $\beta$ are contrast parameters between 0 and 1.

After modulation, the image in the Fourier space becomes

$$
\begin{aligned}
G\left(\nu_{x}, \nu_{y}\right)=F\left(\nu_{x}, \nu_{y}\right)+ & \\
\frac{\alpha}{2} e^{-2 i \pi \phi} & {\left[F\left(\nu_{x}-k_{x}, \nu_{y}-k_{y}\right)+F\left(\nu_{x}+k_{x}, \nu_{y}+k_{y}\right)\right]+} \\
& \frac{\beta}{2} e^{-i \pi \phi}\left[F\left(\nu_{x}-\frac{k_{x}}{2}, \nu_{y}-\frac{k_{y}}{2}\right)+F\left(\nu_{x}+\frac{k_{x}}{2}, \nu_{y}+\frac{k_{y}}{2}\right)\right] .
\end{aligned}
$$

and it is finally filtered by the OTF as $H\left(\nu_{x}, \nu_{y}\right) G\left(\nu_{x}, \nu_{y}\right)$. This result is illustrated in figure 1 for a $1 \mathrm{D}$ signal. 
To the best of our knowledge, all existing approaches $[4,5,6,7,9]$ state that $U_{0}, U_{1}$, and $U_{2}$ (see figure 1) are three unknowns that can be recovered by a linear combination of at least three or more modulated images with the same frequency modulation but different phases $\phi$. Unfortunately, this approach completely disregards that redundancy is introduced, as mentioned by Heintzmann in [17]. It needs at least five images when the zero order is present for one grid orientation, and fifteen raw images for one 2D image.

Indeed, because of the Fourier Hermitian symmetry of the real image, $U_{0}$ and $U_{2}$ are complex conjugates of each other. Moreover, the part $U_{1}$ introduced by the zero order is not an additional unknown. In essence, irrespective of the sinusoidal pattern, only two unknowns can be present inside the raw data: the low frequencies between 0 and $\nu_{c}$, labeled $L F$ in figure 1 and the high frequencies between $\nu_{c}$ and $2 \nu_{c}$, labeled $H F$. The raw data is then a mix of several copies of different frequencies in the range of these two unknowns ( $L F$ and $H F$ ). Based on this observation, we demonstrate that estimating the high-resolution image still involves the resolution of a linear system but with only four datasets for four unknowns in a full 2D dataset. Moreover, every extra modulation with frequency $|\boldsymbol{k}|<\nu_{c}$ does not introduce an extra unknown.

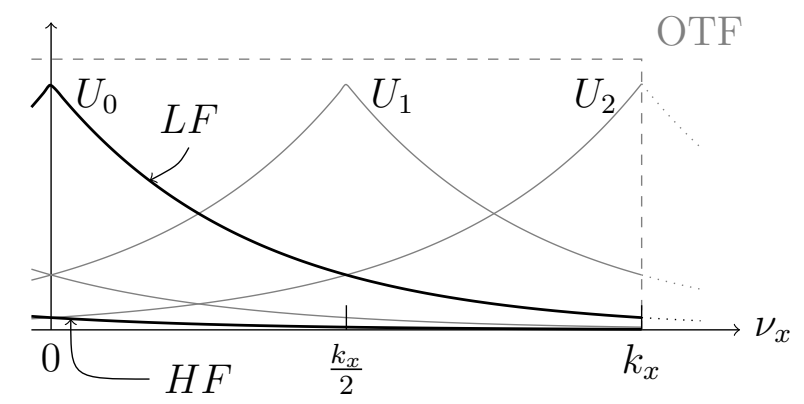

Figure 1: Illustration of amplitude modulation for SIM with zero order. The original spectrum $L F$ is replicated around $\pm \frac{k_{x}}{2}$ and $\pm k_{x}$. The OTF removes all frequencies beyond $\nu_{c}$, equal to $k_{x}$ in this illustration. The unknown $H F$ part comes from the $-k_{x}$ replication while the redundant spectra are in gray.

\section{Data and image model}

\subsection{Forward model}

The unknown image is modeled as $N$ pixels collected in a vector $\boldsymbol{f} \in \mathbb{R}^{N}$. The pixel size is defined to be sufficient to represent all possible frequencies that can be reconstructed from the raw images. The image is illuminated by a light field $\boldsymbol{m}$ modeled as $\boldsymbol{M} \boldsymbol{f}$, where the matrix $\boldsymbol{M}$ is diagonal with $\operatorname{diag}(\boldsymbol{M})=\boldsymbol{m} \in \mathbb{R}^{N}$. The illumination is structured to produce modulations as described by equation (1). Therefore, the vector $\boldsymbol{m}$, or the diagonal of $\boldsymbol{M}$, depends on the five unknown parameters $\boldsymbol{\theta}=\left[k_{x}, k_{y}, \phi, \alpha, \beta\right]$ and corresponds to the fringe pattern. 
Fast myopic 2D-SIM Super Resolution Microscopy with Joint Modulation Pattern Estimation6

The optics and microscope response are supposed to be linearly spatial invariant. The output is obtained by the convolution of the input image $\boldsymbol{M} \boldsymbol{f}$ with a known point spread function (PSF) thanks to simulation of the Airy disc or measurement. Being linear, the convolution operation can be written as $\boldsymbol{C} \boldsymbol{M} \boldsymbol{f}$, where $\boldsymbol{C}$ is a block circulant with circulant block (BCCB) convolution matrix. The BCCB nature of $\boldsymbol{C}$ makes the application of the convolution manageable in Fourier space

$$
C M_{\theta} f=F^{\dagger} \Lambda_{C} F M_{\theta} f
$$

where $\Lambda_{C} \in \mathbb{C}^{N \times N}$ is a diagonal complex matrix with the OTF as the diagonal and $\boldsymbol{F}, \boldsymbol{F}^{\dagger}$ are the unitary forward and reverse Fourier transform matrices, respectively. Equation (3) states that the convolution can be easily computed by filtering in the Fourier domain.

The third element is camera detector integration. We assume the detector as being squared with perfect integration on its surface. The camera integration is modeled as a convolution then sampling at the detector resolution. Next, camera convolution is integrated with the optic convolution, and only sampling remains. This operation is written as a matrix $\boldsymbol{S} \in\{0,1\}^{N \times M}$ with only one 1 per line, 0 otherwise. Concretely $\boldsymbol{S}$ is identity if $N=M$, or make a subsampling by a factor of two if $N=2 M$. In the latter case, the image resolution is doubled with respect to the raw data resolution, and the camera convolution is a $2 \times 2$ square response.

Finally, the full model writes

$$
\boldsymbol{g}_{i}=\boldsymbol{S C} \boldsymbol{M}_{\boldsymbol{\theta}_{i}} \boldsymbol{f}+\boldsymbol{n}_{i}=\boldsymbol{H}_{i} \boldsymbol{f}+\boldsymbol{n}_{i}
$$

where $\boldsymbol{g}_{i} \in \mathbb{R}^{M}$ are $i$-th raw data and $\boldsymbol{n}_{i} \in \mathbb{R}^{M}$ an unknown noise. Then, $I$ raw data are acquired, stacked, and the complete model is written as

$$
g=H f+n
$$

where

$$
\boldsymbol{H}=\left(\begin{array}{ccc}
\boldsymbol{H}_{1} & & \\
& \ddots & \\
& & \boldsymbol{H}_{I}
\end{array}\right)\left(\begin{array}{c}
\boldsymbol{I} \\
\vdots \\
\boldsymbol{I}
\end{array}\right) \quad \text { and } \quad \boldsymbol{n}=\left(\begin{array}{c}
\boldsymbol{n}_{1} \\
\vdots \\
\boldsymbol{n}_{I}
\end{array}\right) .
$$

However, the naive use of this forward model is ill posed because the least square solution of the system

$$
\left(\boldsymbol{H}^{t} \boldsymbol{H}\right) \boldsymbol{f}=\boldsymbol{H}^{t} \boldsymbol{g}
$$

may have several solutions (without additional choice), or is unstable otherwise [18]. This characteristic comes from the sub-sampling $\boldsymbol{S}$ and the convolution $\boldsymbol{C}$, and it is the main reason for the use of Wiener filtering in classical methods [16, 6]. We propose an alternative approach where the image and the modulation parameter models are defined jointly with the forward model owing to a regularized criterion. 


\subsection{Image model}

To eliminate the ill-posed feature of the least square criterion, we introduce a regularization term leading to the mixed criterion

$$
J(\boldsymbol{x})=\|\boldsymbol{g}-\boldsymbol{H} \boldsymbol{f}\|^{2}+\lambda\|\boldsymbol{D} \boldsymbol{f}\|^{2} .
$$

This kind of criterion is well known and has been widely studied and used in various fields and applications $[19,20,21,22,23,18]$. The prior fidelity term $\|\boldsymbol{D} \boldsymbol{f}\|^{2}$ stabilizes the problem and further particular solutions. To counterbalance noise amplification

arising from the data fidelity term, $\boldsymbol{D}$ is chosen as a differential operator or high-pass filter. We then choose the Laplacian second-order differential operator along the lines and columns implemented as the 2D impulse response

$$
d=\left[\begin{array}{ccc}
0 & -1 & 0 \\
-1 & 4 & -1 \\
0 & -1 & 0
\end{array}\right]
$$

Moreover, we implement filtering in Fourier space $\boldsymbol{D}=\boldsymbol{F}^{\dagger} \boldsymbol{\Lambda}_{\boldsymbol{D}} \boldsymbol{F}$, where $\boldsymbol{\Lambda}_{\boldsymbol{D}}$ is diagonal and the corresponding high-pass filter. Owing to Parseval equality, the criterion can then be written as

$$
J(\boldsymbol{x})=\|\boldsymbol{g}-\boldsymbol{H} \boldsymbol{f}\|^{2}+\lambda\left\|\boldsymbol{\Lambda}_{\boldsymbol{D}} \boldsymbol{f}\right\|^{2} .
$$

where $\stackrel{f}{\boldsymbol{f}}=\boldsymbol{F} \boldsymbol{f}$ is the discrete Fourier transform (DFT) of the image. The regularization parameter $\lambda$ determines the balance between the data fidelity term, that tends to introduce high frequencies with noise amplification and instability, and the prior fidelity term that tends to favor image smoothness. Owing to this Tikhonov regularization, the criterion remains quadratic with a fast algorithm such as a conjugate gradient [24] to optimize it. Note that this prior corresponds to the classical Wiener filter. Other prior are possible, such as TV or $\ell_{2}-\ell_{1}$, which would lead to a nonlinear estimator for better image reconstruction with the price of a more complex and slower algorithm.

The choice of the $\lambda$ parameter value is a difficult question, and a significant part of the literature is dedicated to it $[25,21,26,27,15,28,29]$. In this work, the value is considered known and fixed by hand to reconstruct satisfactory images. This possibility relies on the Bayesian framework such as in [15].

\subsection{Modulation parameters model}

The modulation parameters must be estimated from the data jointly with the image. With $I$ raw data acquired, $5 \times I$ parameters must be estimated. Nevertheless, little information is available about them except some interval constraints.

- Concerning the modulation frequencies $\boldsymbol{k}$, the nearest discrete frequencies from the DFT of data can be easily located [9] at indices $(n, m)$. This allows stating that the true frequency $\boldsymbol{k}^{*}=\left(k_{x}^{*}, k_{y}^{*}\right)$ is inside the interval

$$
k_{x}^{*} \in\left[\left(n-\frac{1}{2}\right) \Delta_{x},\left(n+\frac{1}{2}\right) \Delta_{x}\right]
$$


Fast myopic 2D-SIM Super Resolution Microscopy with Joint Modulation Pattern Estimation8

and

$$
k_{y}^{*} \in\left[\left(m-\frac{1}{2}\right) \Delta_{y},\left(m+\frac{1}{2}\right) \Delta_{y}\right]
$$

where $\Delta_{x}$, and $\Delta_{y}$ are the spectral resolution of the DFT.

- The phase value is unknown. Shroff et al [9] guess the phase from the data spectrum phase value at $(n, m)$ frequency. These approaches assume that phases from other replications are negligible and that frequency modulation is exactly at the discrete frequency $\left(n \Delta_{x}, m \Delta_{y}\right)$. Unfortunately, such hypotheses are not available for $2 \mathrm{D}$ super resolution SIM since frequency modulation slightly change with experimental conditions, especially with significant noise. We consider here that the true phase value is located inside the interval

$$
\phi^{*} \in[0,2 \pi] .
$$

- Knowledge about the contrast parameters is also limited. As for the phases, we only use physical information saying that the contrast parameters are inside the interval

$$
\alpha^{*}, \beta^{*} \in[0,1]^{2}
$$

\subsection{Complete model}

The solution to the problem of joint estimation of the image $\boldsymbol{f}$ and the full set $\boldsymbol{\theta}=[\boldsymbol{k}, \boldsymbol{\phi}, \boldsymbol{\alpha}, \boldsymbol{\beta}]$ of $5 \times I$ modulation parameters are chosen as the joint minimizer of constrained regularized least square criterion

$$
\begin{gathered}
\hat{\boldsymbol{f}}, \hat{\boldsymbol{\theta}}=\underset{\boldsymbol{f}, \boldsymbol{\theta}}{\arg \min }\left\|\boldsymbol{g}-\boldsymbol{H}_{\boldsymbol{\theta}} \boldsymbol{f}\right\|^{2}+\lambda\|\boldsymbol{D} \boldsymbol{f}\|^{2} \text { subject to } \\
\boldsymbol{k} \in\left[\boldsymbol{k}_{m}, \boldsymbol{k}_{M}\right] \\
\boldsymbol{\phi} \in[0,2 \pi]^{I} \\
\boldsymbol{\alpha}, \boldsymbol{\beta} \in[0,1]^{2 \times I} .
\end{gathered}
$$

The problem is well posed; the regularization factor $\lambda \boldsymbol{D}^{t} \boldsymbol{D}$ ensures the uniqueness of the solution, stabilizes the inversion, and circumvents noise amplification. The defined image and parameters solution correspond to the values that best reproduce the data $\boldsymbol{g}$ while satisfying the constraints. The criterion is, however, globally non-convex with several local minimizers. Sec. 4 is devoted to an optimisation algorithm that try to reach a satisfactory solution.

From this point, two main questions arise: First, how to compute the solution defined by equation (8)? Second, does this solution solve the initial problem of joint estimation of modulation parameters and image reconstruction ? The next two sections are devoted to the proposed algorithm that computes the solution and to showing results that illustrate the effectiveness of the proposition. 
Fast myopic 2D-SIM Super Resolution Microscopy with Joint Modulation Pattern Estimation9

\section{Myopic SIM image reconstruction}

Several computational difficulties arise in resolving the problem defined by equation (8).

(i) The solution cannot be expressed explicitly. This point is addressed with an alternate block optimization algorithm on $\boldsymbol{\theta}$ and $\boldsymbol{f}$.

(ii) The criterion, w.r.t. the modulation parameters $\boldsymbol{\theta}$, is nonlinear because of the cosine and can have several local minima. Hopefully, the number of parameters is small and, as demonstrated here, global optimization is feasible.

(iii) The dimension, w.r.t. the image $\boldsymbol{f}$, is very large and non-stationary because of the presence of the forward model $\boldsymbol{H}$. Despite the fact that the regularized least square solution is explicit,

$$
\hat{\boldsymbol{f}}=\left(\boldsymbol{H}^{t} \boldsymbol{H}+\lambda \boldsymbol{D D}\right)^{-1} \boldsymbol{H}^{t} \boldsymbol{g}
$$

the problem is too large to invert the matrix and make $\boldsymbol{f}$ directly tractable with (9). The proposed solution relies on the well-known conjugate gradient algorithm to solve large linear systems.

To solve the joint problem, we propose an alternate minimization algorithm 1 that we describe in more detail in the next section. Nevertheless, the quality of the initial image $\boldsymbol{f}^{(0)}$ is an important point. The non-linearity and multi-modality of the criterion make the convergence of line 1.5 dependent on the initial point. In section 5.5.1, we propose a robust and easily feasible initial image.

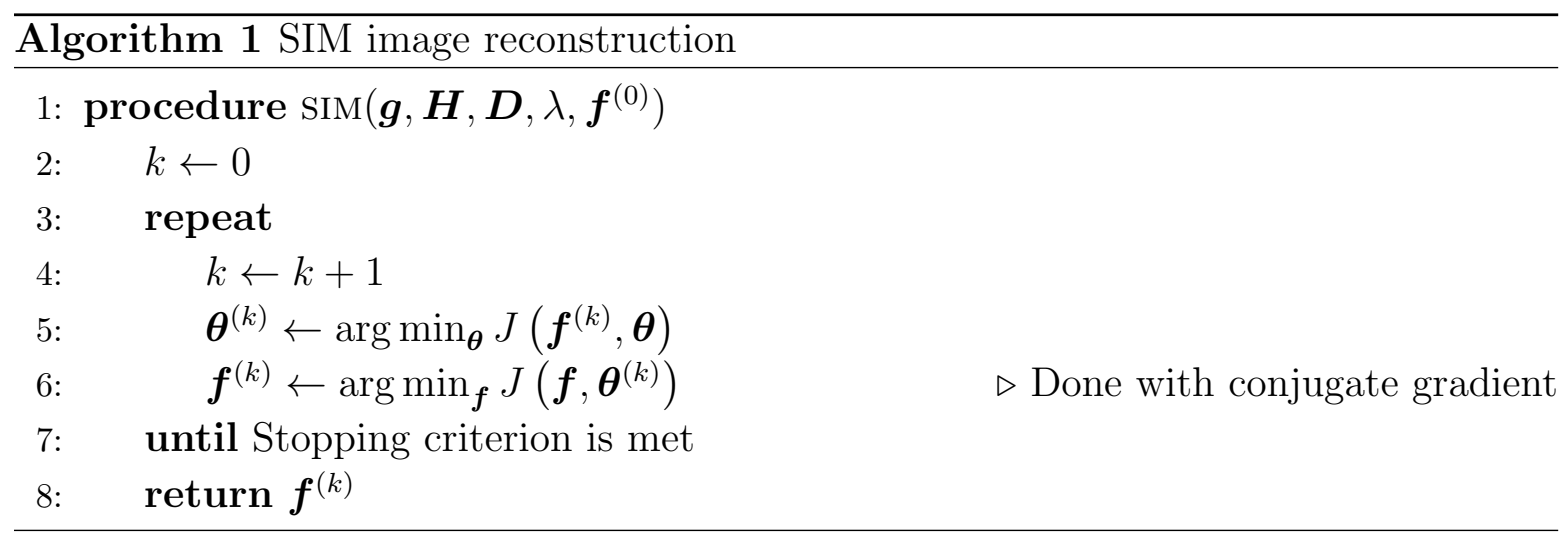

\subsection{Modulation parameters optimization}

The optimization of the modulation parameters is not straightforward, even more so when only four raw data images are available. This step requires finding the parameters $\boldsymbol{\theta}$ that best reproduce the data with a fixed image $\boldsymbol{f}$ with respect to equation (8). However, minor simplifications are possible. First, the regularization term can be removed because it does not depend on $\boldsymbol{\theta}$. Second, the criterion can be split into one criterion for each raw data $\boldsymbol{g}_{i}$ because the modulation parameter model is independent 
when $\boldsymbol{f}$ is fixed. Consequently, the line 5 of the algorithm 1 corresponds to I's parallel optimization of the constrained nonlinear least square criterion

$$
\begin{gathered}
J_{i}\left(\boldsymbol{\theta}_{i}\right)=\left\|\boldsymbol{g}_{i}-\boldsymbol{S H} \boldsymbol{M}_{\boldsymbol{\theta}_{i}} \boldsymbol{f}\right\|^{2} \quad \text { subject to } \\
\left.\boldsymbol{k}_{i} \in\left[\boldsymbol{k}_{m}, \boldsymbol{k}_{M}\right], \quad \phi_{i} \in[0,2 \pi], \quad \alpha_{i}, \beta_{i} \in\right] 0,1\left[^{2}\right.
\end{gathered}
$$

with $\boldsymbol{\theta}_{i}=\left[k_{x i}, k_{y_{i}}, \phi_{i}, \alpha_{i}, \beta_{i}\right]$ and the diagonal elements of $\boldsymbol{M}_{\boldsymbol{\theta}_{i}}$, with $q \in[1, \ldots, N]$,

$$
\begin{aligned}
\boldsymbol{M}_{i}[q]=1+\beta_{i} \cos \left(\pi k_{x, i} x[q]+\pi k_{y, i} y[q]+\phi_{i}\right)+ \\
\quad \alpha_{i} \cos \left(2 \pi k_{x, i} x[q]+2 \pi k_{y, i} y[q]+2 \phi_{i}\right) .
\end{aligned}
$$

This criterion is only the least squares between a "simulator output" and the data, when the modulation parameters are varied.

The optimization scheme of criterion (10) has been established owing to practical observation. First, the contrast parameters $(\alpha, \beta)$ and cosine parameters $(\boldsymbol{k}, \phi)$ form two distinct families of parameters. The estimated value for the cosine parameters can be good enough even if the contrast parameter is wrong but with a significant value such as 0,5 . On the contrary, if the cosine parameters are not good enough, the estimation of the contrast can be extremely low, yielding to 0 value. In other words, if the cosine shape is incorrectly estimated, the best residual is obtained without any modulation. These two observations led us to first estimate the cosine parameters with ad hoc values for the contrast in the first loop of algorithm 1, and to use the contrast parameter as an empirical diagnostic tool, as already suggested in $[5,6]$.

The cosine parameters are more difficult: the criterion is nonlinear because of the cosine, can have several local minima, and the three parameters $\left(k_{x}, k_{y}\right.$, and $\left.\phi\right)$ are strongly correlated. Therefore, we must exhaustively search the full volume in three directions using a global optimization algorithm

$$
\widehat{\boldsymbol{\theta}}=\left\{\boldsymbol{\theta}^{*} \in \mathcal{S} \mid\left\|\boldsymbol{g}-\boldsymbol{H}_{\boldsymbol{\theta}^{*}} \boldsymbol{f}\right\|^{2} \leq\left\|\boldsymbol{g}-\boldsymbol{H}_{\boldsymbol{\theta}} \boldsymbol{f}\right\|^{2}, \forall \boldsymbol{\theta} \in \mathcal{S}\right\}
$$

where $\mathcal{S}$ is a finite set of evaluated points inside $\left[\boldsymbol{k}_{m}, \boldsymbol{k}_{M}\right] \times[0,2 \pi]$. All other tested algorithms (nonlinear conjugate gradient, newton method, and Nealder-Mead for instance), most often local ones, were unable to find the global minimum necessary for good image reconstruction. The advantage of this algorithm is the parallel evaluation of each tested point in $\mathcal{S}$ and the criterion evaluation $J(\boldsymbol{\theta})$ is only required at the current point.

The contrast parameters lead to an easily solved linear least squares with the details explained in Appendix A.

\subsection{Image optimization}

Unlike the modulation parameters, the image optimization step (line 6 of algorithm 1) involves a large but linear and strictly convex problem. The solution equation (9) can 
then be approximated owing to a preconditioned conjugate gradient algorithm that solves the system using the gradient vector

$$
\nabla J(\boldsymbol{f})=2 \boldsymbol{H}^{t}(\boldsymbol{H} \boldsymbol{f}-\boldsymbol{g})+2 \lambda \boldsymbol{D}^{t} \boldsymbol{D} \boldsymbol{f}
$$

that does not involve large matrix inversion. As this step is inside a larger alternate minimization strategy, this subpart can be stopped early. We do not describe in more detail the conjugate gradient because a significant amount of literature is devoted to it $[24,30]$. Appendix B describes a preconditioning matrix that largely diminishes the number of iterations.

\section{Results}

This section shows the results of our myopic SIM approach for experimental and simulated data with only four raw images, with zero order present in the fringe $(\beta \neq 0)$. We compare our results with the classical approach where at least 9 or 15 raw images are needed and show that we provide the same quality as we already demonstrated in [15]. With our method, we can also estimate the modulation parameters. We compare our results to Shroff [9], which is the sole method, to the best of our knowledge, that can estimate only the phase parameters with four images (modulation depth are fixed to the true value in the Shroff tests). This method estimates the frequencies modulation by locating the maximum value pixel, if visible, in Fourier space. Thus, the phase estimate is the phase value at this frequency component and therefore neglects wrong location and noise corruption. The amplitude is also estimated by ratio with the null frequency, corrected by the OTF value. This method is very fast but is not precise and inefficient when the frequency modulation is near the Abbe limit.

The algorithm is initialized with $\boldsymbol{f}^{(0)}$ being the wiener deconvolution [31] of a widefield (image obtained using a uniform illumination) image and $\alpha^{(0)}=\beta^{(0)}=0,5$. The widefield can be a real one if available, or the mean of several modulated images. In the latter case, nine classical images are needed to avoid incorrect convergence, and thus, it is not advisable for our method. In our tests, a widefield image is available. The initial exhaustive grid search is performed with $10 \times 10 \times 40$ points for $\left(k_{x}, k_{y}\right.$, and $\left.\phi\right)$. The search interval is initialized with prior constraints as explained in section 3.3.3 and are reduced by a factor 0,9 after each loop for refinement. The implementation is done with Python and standard library (numpy, ...) and typical computing time is between 2 and 10 minutes.

\subsection{Results on simulated data}

The simulations are conducted using two test images: mire, and boat. For all simulated data, the acquisition scheme is

- one widefield image $(N=M=256 \times 256)$. 
- three modulated images $(I=3)$ with respective grid orientation $\left[0, \frac{2 \pi}{3},-\frac{2 \pi}{3}\right]+\frac{\pi}{4}$, with contrast $\alpha=0,2, \beta=0,8$, and modulation frequency $|\boldsymbol{k}|=0,2$. Figure 2 shows the dataset for the mire test.

- an Airy theoretic OTF with reduced frequency cut $\left|\nu_{c}\right| \approx 0,26$

- white gaussian noise with different levels $\left(\gamma_{\boldsymbol{n}}=10\right.$ and 0,1$)$.

- the frequency modulation is 0.23 in reduced frequency.

- all images are in the same displayed with same dynamics with original image values between 0 and 256 .

The algorithm settings are always the same as described in section 3 and the process takes a few minutes with a $256 \times 256$ pixels image on a $4 \times 1.9 \mathrm{GHz} \mathrm{CPU}$ with $8 \mathrm{~GB}$ of memory. The tests were conducted with the images mire, and boat, and two noise levels with mire and mire-hn. All known methods [4, 5, 6, 32] rely on the cross-correlation of extracted spectra with the classical 9 or 15 images. They are thus inefficient with only four images. We compare the proposed myopic SIM method to Shroff et al [9], which is the sole method that can also provide phase values.
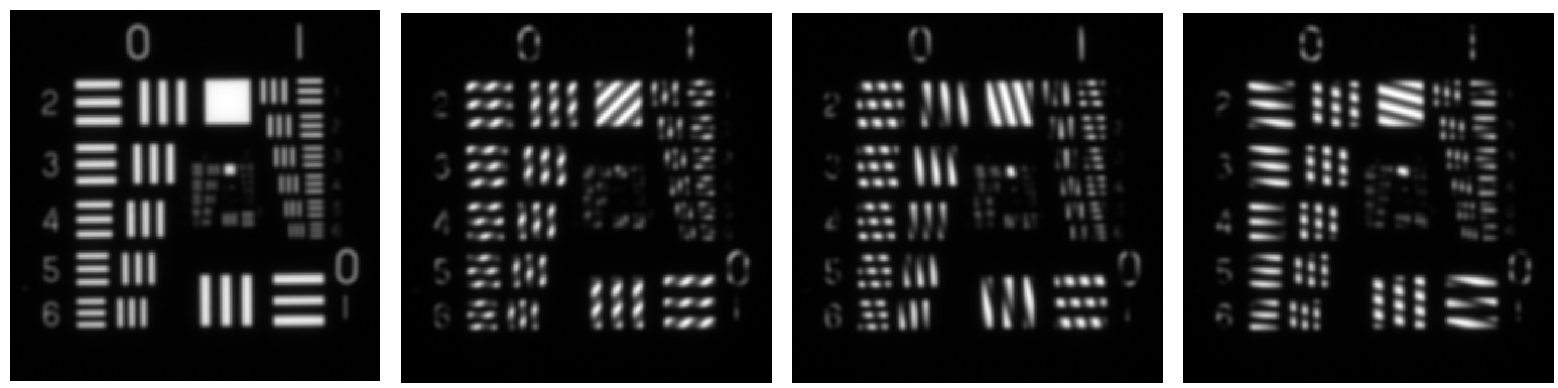

Figure 2: The four raw images used for the myopic SIM reconstruction in the mire test. The first image on the left is the widefield image, and the three other are modulated. The main visible fringes come from the interference between the zero order and the \pm 1 order, with $k_{x} / 2$ frequency.

The figure 3 shows a zoom of the mire results with 3 a being the true image. The figure $3 \mathrm{c}$ is the reconstruction where modulation parameters are estimated with the [9] method. Artifacts are present and make the image difficult to analyze. The power spectral density (PSD, or squared absolute value of the Fourier transform) is illustrated in figure 4c. The PSD exhibits classical interference in SIM because of the wrong estimation of the modulation parameters. On the contrary, figures $3 \mathrm{~d}$ and $4 \mathrm{~d}$ show the results of our myopic SIM approach. The results show an image without visual artifacts and more high frequencies than widefield. The PSD figure $4 \mathrm{~d}$ shows only very limited effect on high frequency. This effect is so small that no differences are visible in comparison with the reconstruction made with true parameter values.

To appreciate the gain in resolution, the figure 5 shows a slice of the image for the widefield and the myopic SIM reconstruction. More details and high frequencies are visible. 
Fast myopic 2D-SIM Super Resolution Microscopy with Joint Modulation Pattern Estimation13

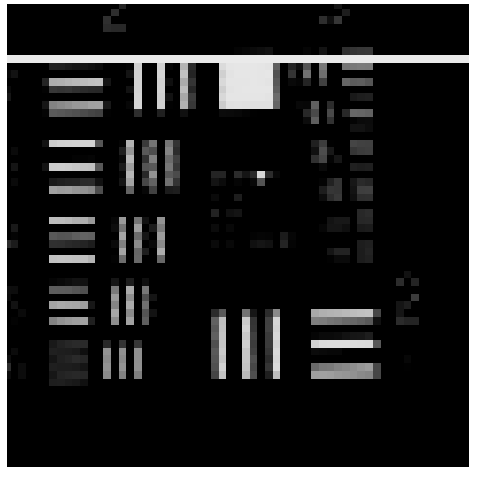

(a) True image

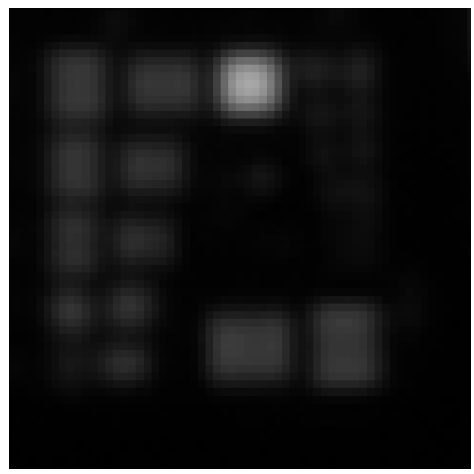

(b) Widefield

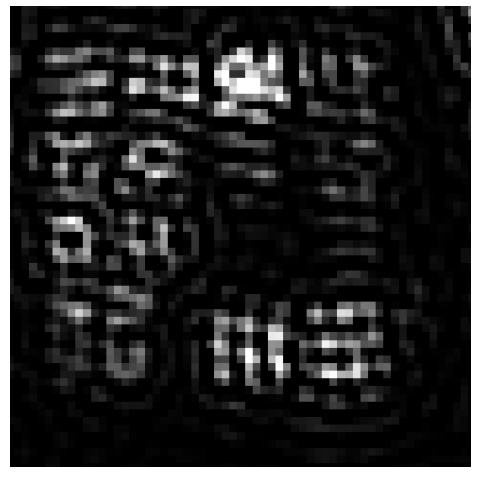

(c) SIM w. [9]

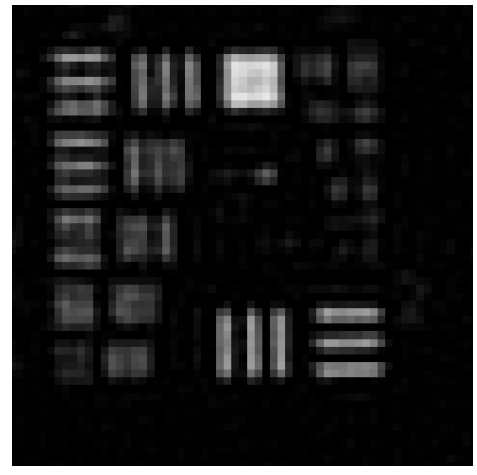

(d) Myopic SIM w. 4 images

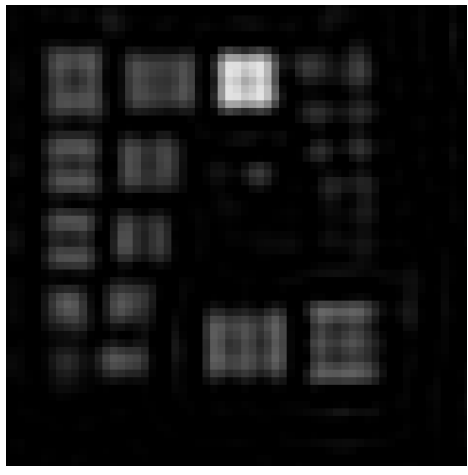

(e) Deconvolution

Figure 3: Zoom on results for the mire test. Figures 3a and 3b show the true image and widefield, respectively. Figure $3 \mathrm{c}$ shows the results with four images with modulation parameters estimated with [9]. Artifacts are strongly present. Figure 3d shows the results of our proposed method. Almost no artifacts are visible. Horizontal and vertical lines are caused by the periodic hypothesis of the DFT algorithm. Figure 3e is the deconvolution of the widefield (Figure 3b) as done in [15] and [31].

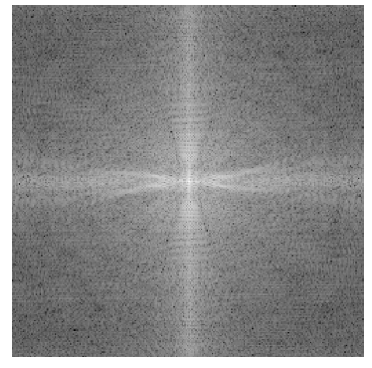

(a) True image

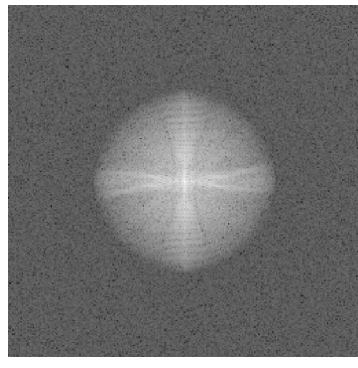

(b) Widefield

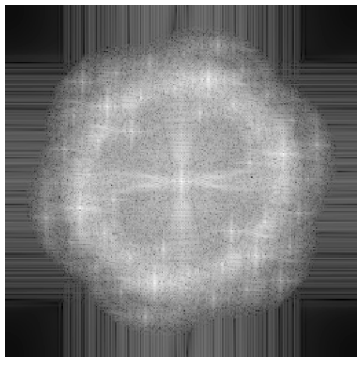

(c) SIM w. [9]

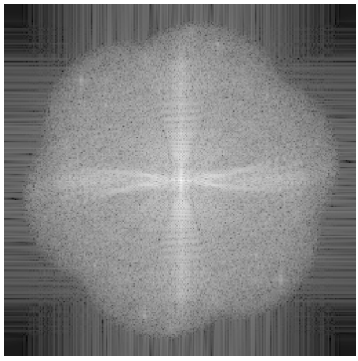

(d) Myopic SIM

Figure 4: Results for the mire test. Figures $4 \mathrm{a}$ and $4 \mathrm{~b}$ show the true image and widefield module spectrum, respectively. Figure 4c shows the results with four images with modulation parameters estimated with [9]. Complex interference patterns are clearly visible. Figure $4 \mathrm{~d}$ shows the results of our proposed method. Almost no artifacts are visible. 


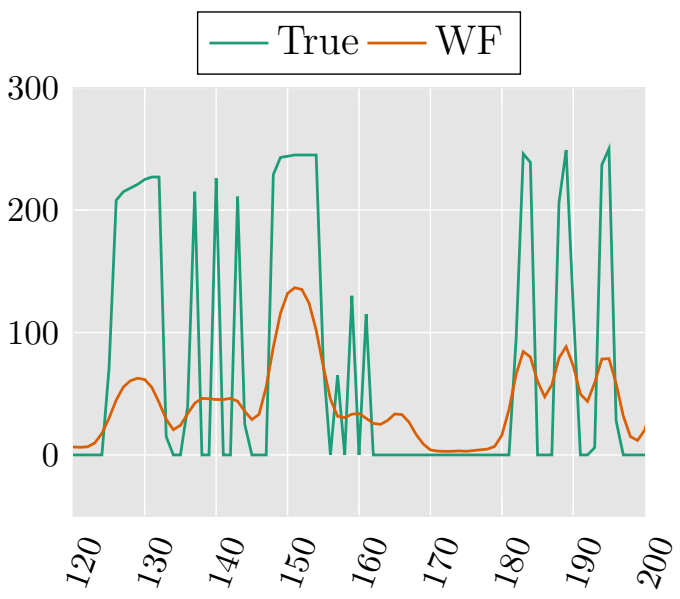

(a) The widefield (WF) comparison

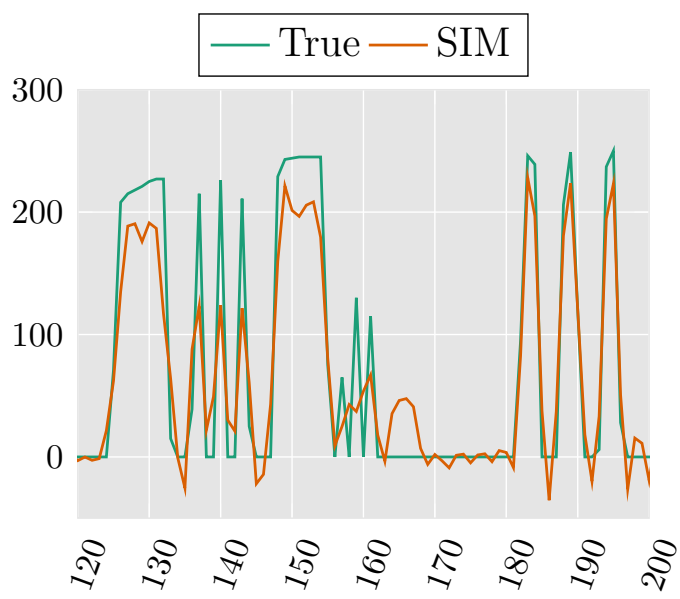

(b) The myopic SIM comparison

Figure 5: Slice comparison for mire test between the true image, the widefield, and the myopic SIM reconstruction. Figure 5a shows the widefield and the true image. We see that the finer details are no longer visible such as around 140. Figure 5b shows that myopic SIM can estimate these originally lost frequencies.

Table 1 shows the error of the estimated parameter values w.r.t. the true values, for the Shroff et al [9] method and our myopic SIM method. We see that, both in absolute and relative error, our method has better estimation by between one and two orders of magnitude. This explains the quality of the reconstructed images. The level of required precision illustrates the difficulty of the problem.

Figure 6 shows the comparison between two reconstructions with two different levels of noise. The mire-hn test has been simulated with 10 times more noise (that is $\gamma_{\boldsymbol{n}}=0.1$ and $\left.\gamma_{\boldsymbol{n}}=100\right)$ and the myopic method is robust and can reconstruct a good high-resolution image without artifacts. However, the reconstructed image quality for mire-hn is obviously slightly lower than expected because more noise affects the measurements.

Finally, the myopic SIM method has been tested with boat image. The figure 7 shows the original image on the left, then the widefield, the SIM reconstruction with parameters estimated with [9], and our myopic SIM method. Again, the proposed approach is effective with good parameter estimation and good image reconstruction, without visible artifacts. The figure 8 shows a slice of the image and illustrates the gain in detail.

\subsection{Results on experimental data}

Tests have been conducted on real microscopic biological data. The setup has already been described in several references such as [33] or [15]. Images are obtained on living HeLa cells where mitochondria are labeled with Mitotracker green, and observed with a $96 \mathrm{X} 1,2 \mathrm{NA}$ objective lens $(N=1014 \times 1024, M=512 \times 512)$ and $I=3)$. The 
Fast myopic 2D-SIM Super Resolution Microscopy with Joint Modulation Pattern Estimation15

\begin{tabular}{|c|c|c|c|c|c|}
\hline \multicolumn{2}{|c|}{$k_{x}$ abs. error } & \multicolumn{2}{|c|}{$k_{y}$ abs. error } & \multicolumn{2}{|c|}{$\phi$ abs. error } \\
\hline$[9]$ & Myopic & {$[9]$} & Myopic & {$[9]$} & Myopic \\
\hline $8 \cdot 10^{-4}$ & $1 \cdot 10^{-5}$ & $8 \cdot 10^{-4}$ & $2 \cdot 10^{-5}$ & 1 & $1 \cdot 10^{-2}$ \\
\hline $1 \cdot 10^{-3}$ & $1 \cdot 10^{-6}$ & $2 \cdot 10^{-3}$ & $2 \cdot 10^{-6}$ & $6 \cdot 10^{-1}$ & $2 \cdot 10^{-3}$ \\
\hline $2 \cdot 10^{-3}$ & $2 \cdot 10^{-5}$ & $1 \cdot 10^{-3}$ & $1 \cdot 10^{-6}$ & $7 \cdot 10^{-1}$ & $7 \cdot 10^{-3}$ \\
\hline
\end{tabular}

(a) Absolute error of modulation parameters for mire test

\begin{tabular}{|c|c|c|c|c|c|}
\hline \multicolumn{2}{|c|}{$k_{x}$ rel. error $(\%)$} & \multicolumn{2}{|c|}{$k_{y}$ rel. error $(\%)$} & \multicolumn{2}{|c|}{$\phi$ rel. error (\%) } \\
\hline$[9]$ & Myopic & {$[9]$} & Myopic & {$[9]$} & Myopic \\
\hline $6 \cdot 10^{-1}$ & $9 \cdot 10^{-3}$ & $6 \cdot 10^{-1}$ & $1 \cdot 10^{-2}$ & & \\
\hline 2 & $2 \cdot 10^{-3}$ & $9 \cdot 10^{-1}$ & $1 \cdot 10^{-3}$ & 27 & $1 \cdot 10^{-1}$ \\
\hline $9 \cdot 10^{-1}$ & $1 \cdot 10^{-2}$ & 2 & $2 \cdot 10^{-3}$ & 33 & $4 \cdot 10^{-1}$ \\
\hline
\end{tabular}

(b) Relative error of modulation parameters for mire test

Table 1: Absolute and relative errors of modulation parameters for the mire test and the three modulated images. Two estimations are compared: the method of Shroff et al [9] and the proposed myopic. The results show that our method improves the reconstruction of between one and two order of magnitude w.r.t. [9]

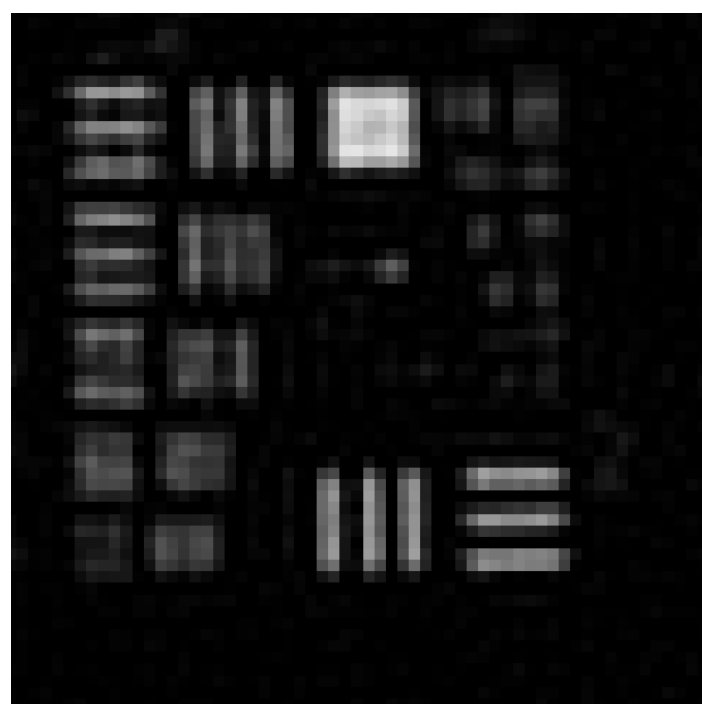

(a) mire reconstruction

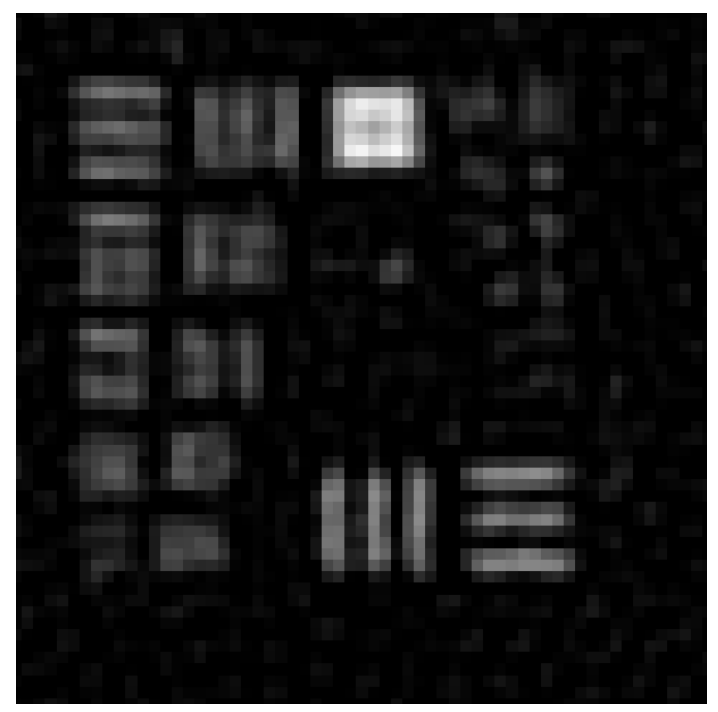

(b) mire-hn reconstruction

Figure 6: Image reconstruction results with the proposed myopic method for mire and mire-hn tests. Figures show good reconstruction results for mire-hn even with 10 times more noise. 


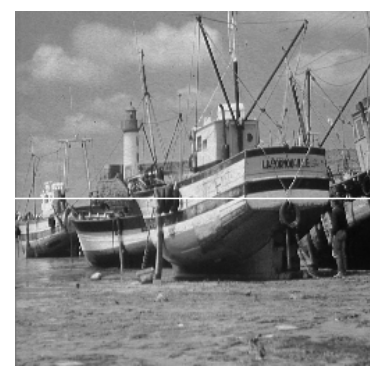

(a) Boat

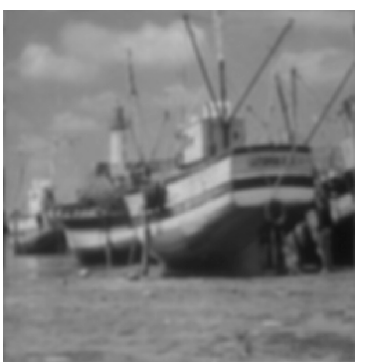

(b) Widefield

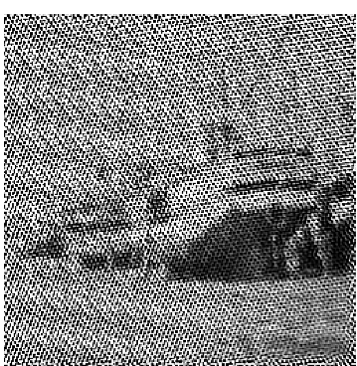

(c) SIM w. [9]

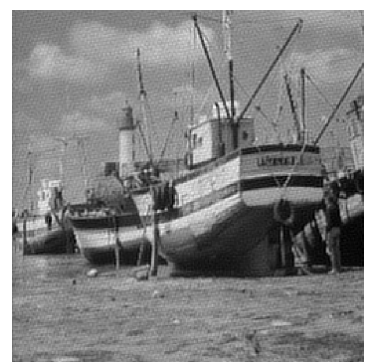

(d) Myopic SIM

Figure 7: The result for boat tests.

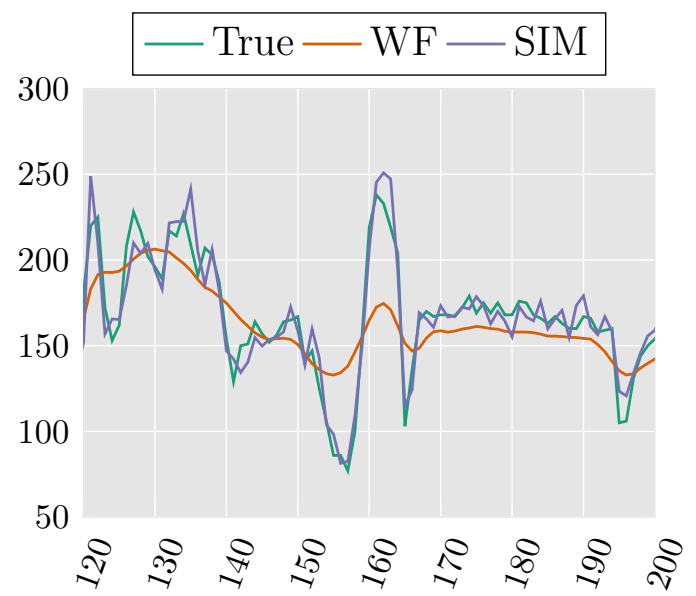

(a) boat slice

Figure 8: Slice comparison for boat test between the true image, the widefield, and the myopic SIM reconstruction. The results clearly show that high frequencies are recovered w.r.t. to the widefield.

illumination pattern was generated by three-beam interference and the SIM image was obtained from four raw images. The exposure time is $500 \mathrm{~ms}$ per raw image. Figures $9 \mathrm{~b}$ and 9a, respectively, show the classical uniform image and the four-image $(I=3)$ SIM reconstruction using the myopic SIM method. The final obtained resolution is related to the optical setup and the chosen frequency modulation $\boldsymbol{k}$, but an improvement in spatial resolution is clearly visible without visual artifacts while we observe a thick sample.

\section{Conclusion}

We propose a new myopic SIM approach to jointly estimate the modulation parameters with significant precision with a high-resolution image, allowing reconstruction without visible artifacts even with only four raw images. The demonstration has been shown in simulated data and biological samples.

We demonstrated that inverse methodological data processing methods have a great impact on imaging capability in SIM by increasing the frame rate by a factor of $225 \%$ 


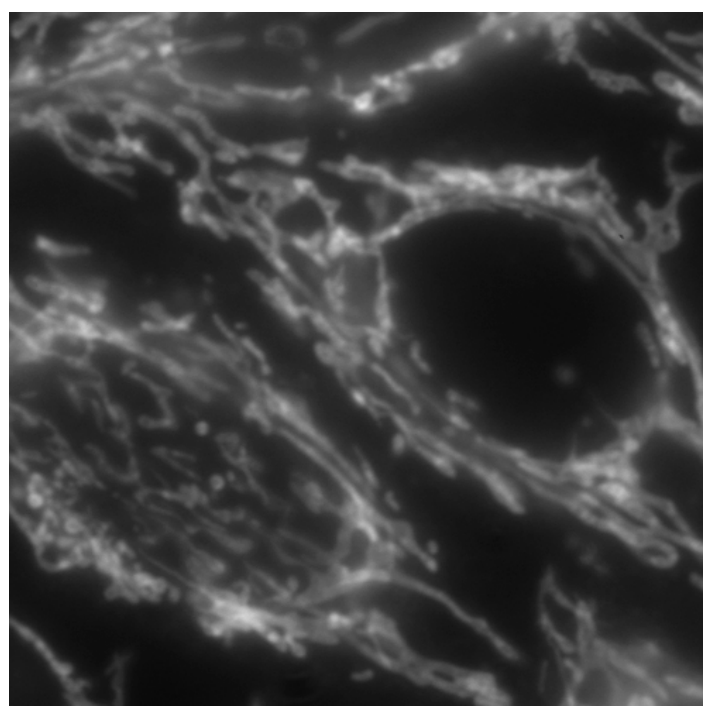

(a) Widefield

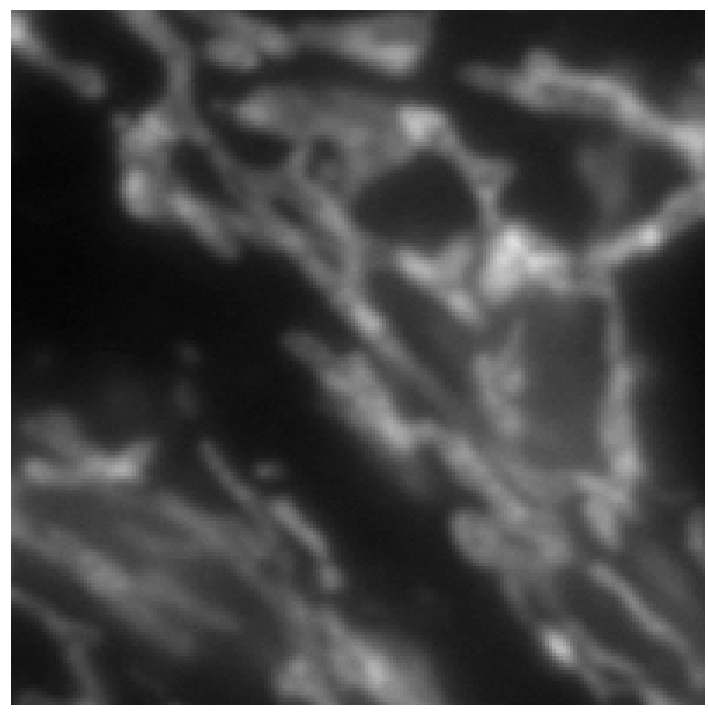

(c) Zoom on widefield

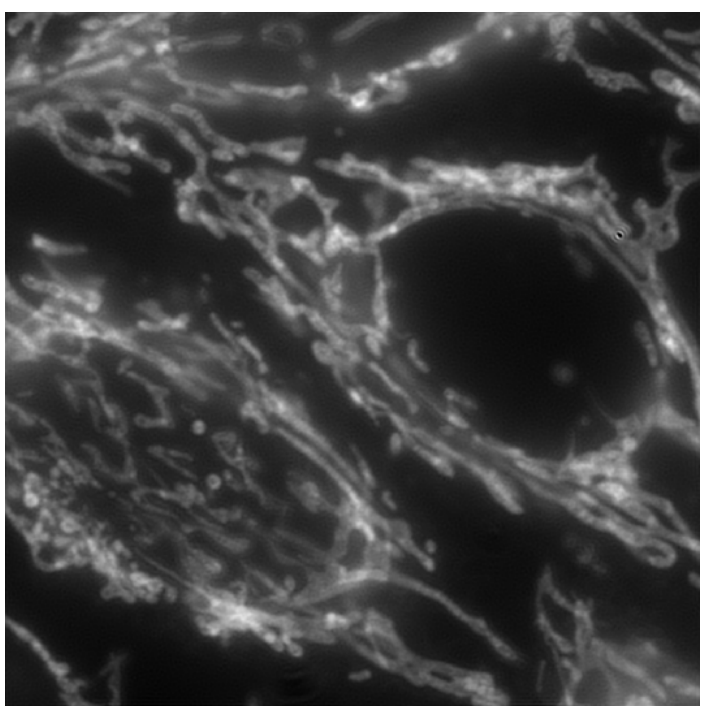

(b) SIM w. 4 images

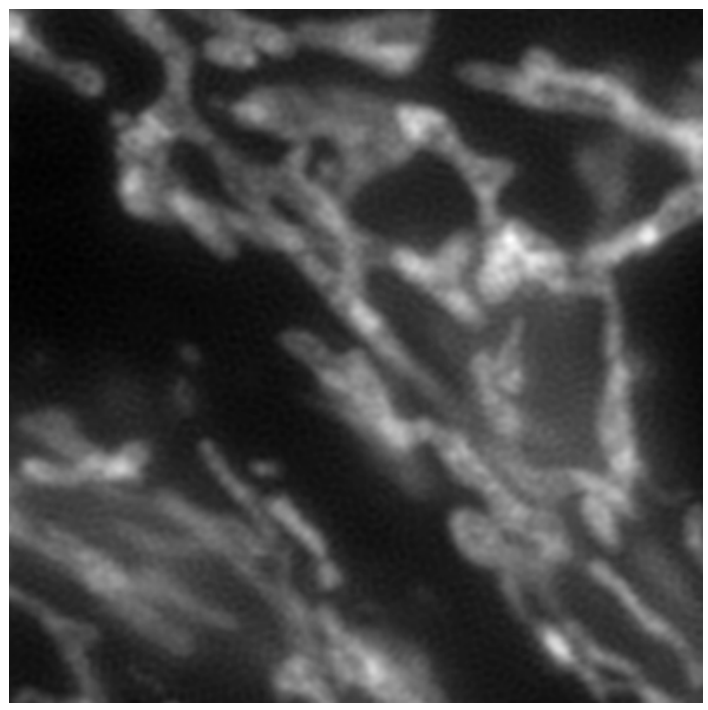

(d) Zoom on SIM w. 4 images

Figure 9: Thick sample data with 96X lens, and marked mitochondria. The exposure time of the widefield is equivalent to that for the four SIM raw data, in the presence of the zero order for the modulation. Spatial resolution enhancement is clearly visible.

(from 9 to 4 images) up to $375 \%$ (from 15 to 4 images), or by the reduction of the number of raw images needed for one reconstruction by a factor of two. Moreover, our estimator allows optical setup simplification by removing the need to block the zero order. Furthermore, the algorithm is independent of the fringe pattern.

Perspective applications are numerous, such as fast 3D imaging. One limitation is the nonstationary noise because of the Poisson nature of the flux. We also might consider out-of-focus background estimation with work first published in [33]. Strong out-of-focus light may modulation parameters to diffucult to estimate without proper handle. Hyper- 
Fast myopic 2D-SIM Super Resolution Microscopy with Joint Modulation Pattern Estimation18

parameter estimation, as in [15], is a possibility with modulation parameters estimation done with a Metropolis-Hastings step for instance. Finally, the use of optimized or more specialized optimization algorithm and implementation could drastically reduce the computation time.

\section{Acknowledgments}

The authors would like to sincerely thank Stéphanie Bonneau, assistant professor of Université Pierre et Marie Curie (UPMC), associated with the Laboratoire Jean Perrin (CNRS - UMPC), for providing the HeLa cells.

\section{Appendix A. Contrast estimation}

The contrast estimation, for one raw image and without the constraints, leads to the minimization of

$$
J\left(\theta_{i}\right)=\left\|\boldsymbol{g}_{i}-\boldsymbol{S C} \boldsymbol{M}_{\theta_{i}} \boldsymbol{f}\right\|^{2}
$$

where $\theta_{i}=\alpha_{i}$ or $\beta_{i}$ and the other parameters are fixed. The modulation matrix $\boldsymbol{M}_{\theta_{i}}$ can be written as

$$
\boldsymbol{M}_{\theta_{i}}=\boldsymbol{I}+\alpha_{i} \boldsymbol{K}_{k_{x}, k_{y}, \phi}+\beta_{i} \boldsymbol{K}_{k_{x}, k_{y}, \phi}^{\prime}
$$

where $\boldsymbol{I}$ is the identity matrix and

$$
\begin{gathered}
\boldsymbol{K}=\cos \left(\pi k_{x} x+\pi k_{y} y+\phi\right) \\
\boldsymbol{K}^{\prime}=\cos \left(2 \pi k_{x} x+2 \pi k_{y} y+\phi\right) .
\end{gathered}
$$

This simple observation of linearity w.r.t. $\alpha$ or $\beta$ allows obtaining an explicit minimizer of

$$
\begin{aligned}
J\left(\theta_{i}\right) & =\left\|\boldsymbol{g}_{i}-\boldsymbol{S} \boldsymbol{C} \boldsymbol{M}_{\theta_{i}} \boldsymbol{f}\right\|^{2} \\
& =\left\|\boldsymbol{g}_{i}-\boldsymbol{S C} \boldsymbol{f}-\alpha_{i} \boldsymbol{S C K} \boldsymbol{f}-\beta_{i} \boldsymbol{S C} \boldsymbol{K}^{\prime} \boldsymbol{f}\right\|^{2}
\end{aligned}
$$

and therefore,

$$
\begin{aligned}
& \hat{\alpha}_{i}=\underset{\alpha_{i}}{\arg \min } J\left(\alpha_{i}\right)=\frac{\tilde{\boldsymbol{g}}_{i}^{t} \tilde{\boldsymbol{f}}}{\|\tilde{\boldsymbol{f}}\|^{2}}
\end{aligned}
$$

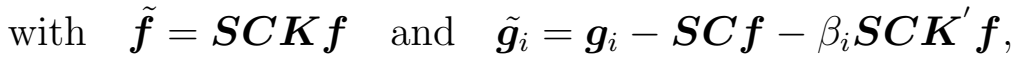

and

$$
\hat{\beta}_{i}=\underset{\beta_{i}}{\arg \min } J\left(\beta_{i}\right)=\frac{\tilde{\boldsymbol{g}}_{i}{ }^{t} \tilde{\boldsymbol{f}}}{\|\tilde{\boldsymbol{f}}\|^{2}}
$$

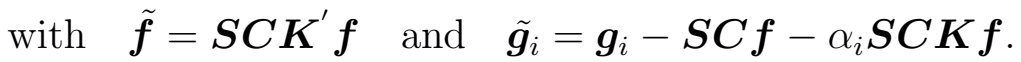




\section{Appendix B. Preconditionner for conjugate gradient}

The goal of a preconditioning matrix is to be the best possible approximation of the Hessian matrix while being easily invertible. The Hessian matrix of problem (7) is

$$
\boldsymbol{H}_{e}=\boldsymbol{H}^{t} \boldsymbol{H}+\lambda \boldsymbol{D}^{t} \boldsymbol{D} .
$$

The $\boldsymbol{H}^{t} \boldsymbol{H}$ matrix is a block matrix composed of blocks such as

$$
\boldsymbol{H}_{c}=\boldsymbol{M}_{\boldsymbol{\theta}_{i}}^{t} \boldsymbol{C}^{t} \boldsymbol{S}^{t} \boldsymbol{S} \boldsymbol{C} \boldsymbol{M}_{\boldsymbol{\theta}_{i}}
$$

that forbids the inversion of the full matrix, as well as factorization in Fourier space. However, the $\boldsymbol{M}_{\boldsymbol{\theta}}$ matrix is diagonal with fluctuations around 1 coming from the modulation and can thus be approximated by the identity matrix. The Hessian matrix blocks can then be approximated by

$$
\boldsymbol{H}_{c} \approx \boldsymbol{C}^{t} \boldsymbol{S}^{t} \boldsymbol{S C} .
$$

Moreover, it appears that the sub-sampling matrix $\boldsymbol{S}^{t} \boldsymbol{S}$ is composed of 1 or 0 on the diagonal. An additional approximation is feasible

$$
\boldsymbol{H}_{c} \approx \boldsymbol{C}^{t} \boldsymbol{C}
$$

where only the convolution part remains. Finally, the Hessian matrix can be approximated by

$$
\boldsymbol{H}_{e} \approx\left(\begin{array}{lll}
\boldsymbol{C}^{t} \boldsymbol{C} & & \\
& \ddots & \\
& & \boldsymbol{C}^{t} \boldsymbol{C}
\end{array}\right)+\lambda \boldsymbol{D}^{t} \boldsymbol{D},
$$

easily factorized in Fourier space

$$
\boldsymbol{H}_{e} \approx \boldsymbol{F}^{\dagger}\left(\left|\boldsymbol{\Lambda}_{\boldsymbol{C}}\right|^{2}+\lambda\left|\boldsymbol{\Lambda}_{\boldsymbol{D}}\right|^{2}\right) \boldsymbol{F}
$$

where $\boldsymbol{F}$ is the matrix Fourier transform, with the property $\boldsymbol{F}^{-1}=\boldsymbol{F}^{\dagger}$, with $\boldsymbol{\Lambda}_{C}$ and $\Lambda_{D}$ as complex diagonal matrices with the transfer function of the optics and the regularization operator, respectively, on the diagonal.

This approximation has an inverse easily tractable

$$
\boldsymbol{M} \approx \boldsymbol{F}^{\dagger}\left(\left|\boldsymbol{\Lambda}_{\boldsymbol{C}}\right|^{2}+\lambda\left|\boldsymbol{\Lambda}_{\boldsymbol{D}}\right|^{2}\right)^{-1} \boldsymbol{F}
$$

and can be used in a preconditioned conjugate algorithm. In practice, a strong improvement in the number of iterations to reach the optimum is observed.

\section{References}

\section{References}

[1] Vivien Marx. "Is super-resolution microscopy right for you?" In: Nature Methods 10.12 (2013), pp. 1157-1163. ISSN: 1548-7091. 
[2] Stefan W. Hell. "Microscopy and its Focal Switch." In: Nature methods 6.1 (2009), pp. 24-32. ISSN: 1548-7105.

[3] Fabian Bergermann et al. "2000-fold parallelized dual-color STED fluorescence nanoscopy." EN. In: Optics express 23.1 (2015), pp. 211-23. ISSN: 1094-4087.

[4] D. Li et al. "Extended-resolution structured illumination imaging of endocytic and cytoskeletal dynamics". In: Science 349.6251 (2015). ISSN: 0036-8075.

[5] Kai Wicker et al. "Phase optimisation for structured illumination microscopy". In: Optics Express 21.2 (2013), p. 2032. ISSN: 1094-4087.

[6] Liisa M Hirvonen et al. "Structured illumination microscopy of a living cell." In: European biophysics journal : EBJ 38.6 (2009), pp. 807-12. ISSN: 1432-1017.

[7] Lin Shao et al. "Super-resolution 3D microscopy of live whole cells using structured illumination". In: Nature Methods october (2011), pp. 2-6. ISSN: 1548-7091.

[8] L. H. Schaefer, D. Schuster, and J. Schaffer. "Structured illumination microscopy: artefact analysis and reduction utilizing a parameter optimization approach". In: Journal of Microscopy 216.May (2004), pp. 165-174.

[9] Sapna A. Shroff, James R. Fienup, and David R. Williams. "Phase-shift Estimation in Sinusoidally Illuminated Images for Lateral Superresolution". In: Journal of the Optical Society of America. sr. A. 26.2 (2009), pp. 413-424.

[10] E. Mudry et al. "Structured illumination microscopy using unknown speckle patterns". In: Nature Photonics 6.5 (2012), pp. 312-315. ISSN: 1749-4885.

[11] Aurélie Jost et al. "Optical Sectioning and High Resolution in Single-Slice Structured Illumination Microscopy by Thick Slice Blind-SIM Reconstruction". In: PLOS ONE 10.7 (2015). Ed. by Vadim E. Degtyar. ISSN: 1932-6203.

[12] R. Ayuk et al. "Structured illumination fluorescence microscopy with distorted excitations using a filtered blind-SIM algorithm". In: Optics Letters 38.22 (2013), p. 4723. ISSN: 0146-9592.

[13] Simon Labouesse et al. "Blind fluorescence structured illumination microscopy: A new reconstruction strategy". In: GRETSI. Lyon, 2015, pp. 2-5.

[14] Siyuan Dong et al. "Resolution doubling with a reduced number of image acquisitions." EN. In: Biomedical optics express 6.8 (2015), pp. 2946-52. ISSN: 2156-7085.

[15] F. Orieux et al. "Bayesian Estimation for Optimized Structured Illumination Microscopy". In: Image Processing, IEEE Trans. on 21.2 (2012), pp. 601-614.

[16] M. G. L. Gustafsson. "Surpassing the lateral resolution limit by a factor of two using structured illumination microscopy". In: Journal of Microscopy 198.2 (2000), pp. 82-87. ISSN: 00222720.

[17] Rainer Heintzmann. "Saturated patterned excitation microscopy with twodimensional excitation patterns". In: Elsevier 34 (2003), pp. 283-291.

[18] Jérôme Idier, ed. Bayesian Approach to Inverse Problems. ISTE Ltd and John Wiley \& Sons Inc., 2008. 
[19] G. Demoment. "Image reconstruction and restoration: overview of common estimation structures and problems". In: IEEE Transactions on Acoustics, Speech, and Signal Processing 37.12 (1989), pp. 2024-2036. ISSN: 00963518.

[20] F. Soulez, É. Thiébaut, and L. Denis. "Restoration of Hyperspectral Astronomical Data with Spectrally Varying Blur". In: EAS Publications Series 59 (2013), pp. 403-416. ISSN: 1633-4760.

[21] Clément Gilavert, Saïd Moussaoui, and Jérôme Idier. "Efficient Gaussian Sampling for Solving Large-Scale Inverse Problems Using MCMC". In: IEEE Transactions on Signal Processing 63.1 (2015), pp. 70-80.

[22] Christian Labat and Jérôme Idier. Convergence of truncated half-quadratic algorithms using conjugate gradient. Tech. rep. 2006.

[23] Jérôme Idier. "Convex Half-Quadratic Criteria and Interacting Auxiliary Variables for Image Restoration". In: IEEE Transactions on Image Processing 10.7 (2001), pp. 1001-1009.

[24] Jorge Nocedal and Stephen J. Wright. Numerical Optimization. Springer, 2000. ISBN: 9780387303031.

[25] F. Orieux, O. Féron, and J.-F. Giovannelli. "Gradient scan gibbs sampler : an efficient high-dimensional sampler application in inverse problems". In: Acoustics Speech and Signal Processing (ICASSP), 2015 IEEE Int. Conf. on. 2015.

[26] Yuling Zheng. "Algorithmes bayésiens variationnels accélérés et applications aux problèmes inverses de grande taille". PhD thesis. Université Paris-Sud, 2014.

[27] Orieux, F. et al. "Estimating hyperparameters and instrument parameters in regularized inversion Illustration for Herschel/SPIRE map making". In: $A \& A$ 549.A83 (2013).

[28] F. Orieux, O. Féron, and J.-F Giovannelli. "Sampling high-dimensional Gaussian distributions for general linear inverse problems". In: Signal Processing Letters, IEEE 19.5 (2012), pp. 251-254.

[29] S. Derin Babacan, Rafael Molina, and Aggelos K. Katsaggelos. "Variational Bayesian Super Resolution". In: IEEE Transactions on Image Processing 20.4 (2011), pp. 984-999.

[30] Jonathan Richard Shewchuk. An Introduction to the Conjugate Gradient Method Without the Agonizing Pain. Tech. rep. Carnegie Mellon University, 1994.

[31] F. Orieux, J.-F. Giovannelli, and T. Rodet. "Bayesian estimation of regularization and PSF parameters for Wiener-Hunt deconvolution". In: Journal of the Optical Society of America A 27.7 (2010), pp. 1593-1607.

[32] Mats G. L. Gustafsson et al. "Three-dimensional resolution doubling in wide-field fluorescence microscopy by structured illumination." In: Biophysical journal 94.12 (2008), pp. 4957-70. ISSN: 1542-0086. 
[33] P. Vermeulen et al. "Out of focus background subtraction for fast structured illumination super resolution microscopy of optically thick samples". In: Journal of Microscopy (2015). 